\title{
Refinements in Brow Reconstruction: Synergy Between Plastic Surgery and Aesthetic Medicine
}

\author{
Silvia Scevola, MD, PhD, Giovanni Nicoletti, MD, FEBoPRAS,1,2 \\ Fabio Randisi, $\mathrm{MD},{ }^{2}$ and Angela Faga, MD, $\mathrm{FICS}^{1,2}$
}

\begin{abstract}
Objective: The purpose of this study was the introduction in clinical practice of the combined application of traditional reconstructive surgery and intense pulsed light (IPL) for the restoration of a post-traumatic eyebrow defect. Case: The authors present a case of surgical eyebrow reconstruction with an axial temporal hair-bearing scalp island flap. A persistent localized erythema and a marked difference in hair density in comparison with the contralateral one harmed the eventual surgical outcome. Results: A combined sequential treatment with IPL achieved both hair density and skin erythema reduction, and eventually allowed for a very satisfactory result. Conclusions: Our experience demonstrated the efficacy of the integrated multidisciplinary approach involving traditional plastic reconstructive surgery and the innovative new technologies in aiming for perfection.
\end{abstract}

\section{Introduction}

$\mathbf{R}$ ESTORATION OF BODY FUNCTION AND APPEARANCE is the basic mission of a plastic surgeon. Nowadays there are an ever increasing number of different tools in the plastic surgeon's arsenal. These range from the traditional surgical techniques to the less invasive aesthetic medical procedures, and are available to address both functional and aesthetic problems. Time-honored and reliable surgical techniques for eyebrow reconstruction include tissue transfers from hairbearing donor sites: full thickness skin grafts, mini- or microscalp or eyebrow hair grafts, flaps from a thick ipsilateral or contralateral eyebrow, and axial skin island flap from the temporal scalp. ${ }^{1-4}$ Intense pulsed light (IPL) is a versatile tool used for treatment of a variety of clinical conditions: hypertrichosis and hirsutism, skin vascular lesions (port wine stains, rosacea, teleangectasias, erythrosis), acne, and skin aging. ${ }^{5-13}$

This case report presents an interesting combined application of traditional reconstructive surgery and IPL for the restoration of a post-traumatic eyebrow defect.

\section{Case Report}

A 28-year-old male patient was admitted to our Plastic Surgery Department for a post- traumatic loss of the lateral half of his left eyebrow, which had occurred 1 year previously.

The clinical examination demonstrated a broad area of retracting scar tissue involving his left forehead, with complete loss of the lateral half of his ipsilateral eyebrow (Fig. 1).

Surgical eyebrow reconstruction was performed, with an axial temporal-hair bearing scalp island flap pedicled on the anterior branch of the superficial temporal artery and venae comitantes. The flap was tailored on the contralateral brow and transferred to the recipient site through a subcutaneous tunnel, and the donor site was repaired with a local rotation scalp flap (Figs. 2 and 3). Two months later, multiple Z-plasties were performed to refine the alignment between the native medial eyebrow and the scalp hair-bearing island flap (Figs. 4-6). The patient regularly trimmed hair growth in the reconstructed eyebrow, and this commitment did not give rise to any complaint by him. Nevertheless, although a significant improvement had been achieved with the restoration of the missing brow tail, the patient still complained of an overall unnatural eyebrow appearance. Objectively, a persistent localized erythema and a marked difference in hair density in comparison with both the native medial half of the left eyebrow and the contralateral one was still apparent 9 months after the last surgical step (Fig. 7).

In order to address such residual imperfections, an IPL treatment was planned using the StarLux ${ }^{\circledR} 500$ IPL platform from Palomar. The LUX RS ${ }^{\mathrm{TM}}$ hand piece was employed for hair removal (wavelength $650-1200 \mathrm{~nm}$, spot size $12 \times 28 \mathrm{~mm}$, pulse length $5-400 \mathrm{~ms}$, maximal fluence $70 \mathrm{~J} / \mathrm{cm}^{2}$, maximal frequency $2 \mathrm{~Hz}$ ). Thirty-six $\mathrm{J} / \mathrm{cm}^{2}$ of energy were delivered through four $12 \times 28 \mathrm{~mm}$ spots for $20 \mathrm{msec}$ per spot. The

\footnotetext{
${ }^{1}$ University of Pavia, Advanced Technologies for Regenerative Medicine and Inductive Surgery Research Centre, Pavia, Italy.

${ }^{2}$ Plastic and Reconstructive Surgery Unit, University of Pavia, Salvatore Maugeri Research and Care Institute, Pavia, Italy.
} 


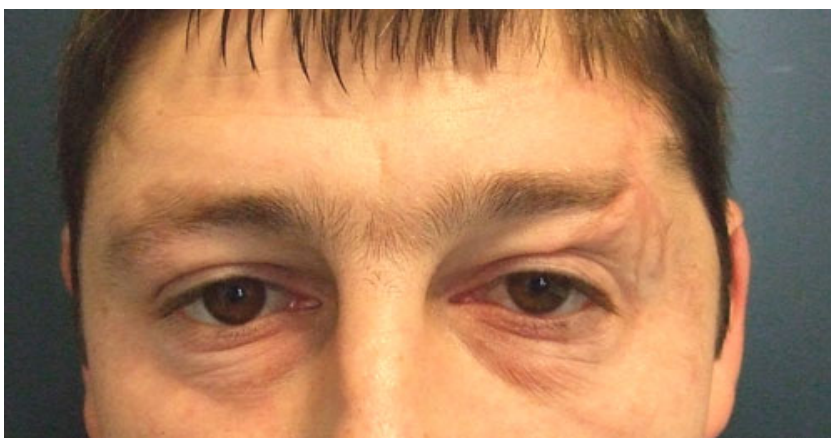

FIG. 1. Pre-treatment view.

MAXG ${ }^{\mathrm{TM}}$ hand piece was employed for scar erythema reduction (wavelength 500-670 and $870-1200 \mathrm{~nm}$, spot size $10 \times 15 \mathrm{~mm}$, pulse length $1-500 \mathrm{~ms}$, maximal fluence $80 \mathrm{~J} / \mathrm{cm}^{2}$, maximal frequency $2 \mathrm{~Hz}$ ). Fifty $\mathrm{J} / \mathrm{cm}^{2}$ of energy were delivered through eight $10 \times 15 \mathrm{~mm}$ spots for $40 \mathrm{msec}$ per spot. This dual combination treatment was repeated after 2 months in order to address some residual imperfections. The parameters were the same as at the first session, except that the number of spots was decreased to two for the LUX RS hand piece and to six for the MAXG hand piece.

Over a period of 6 months, a significant improvement in the overall clinical picture was noted, with a subtotal reduction of scar erythema and a hair density and distribution in the skin scalp island flap similar to that in the native eyebrow (Fig. 8). This outcome was considered to be very satisfactory by both the surgeons and the patient

\section{Discussion}

A plastic surgeon is committed to restoration of both body function and the aesthetic appearance of beauty. Several surgical techniques have been described for eyebrow recon-

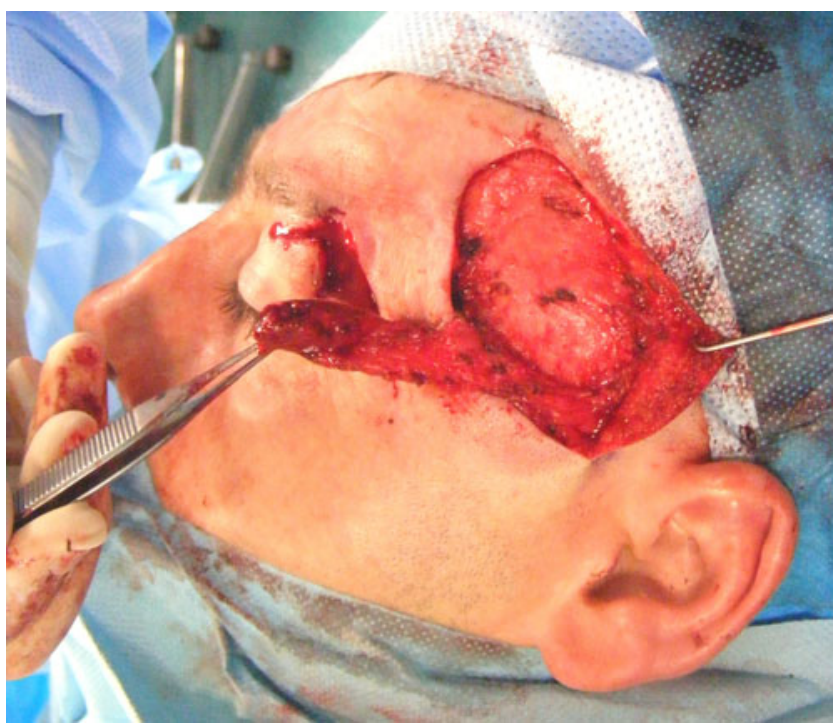

FIG. 2. First surgical stage intra-operative view: the axial temporal hair-bearing scalp island flap pedicled on the anterior branch of the superficial temporal artery and venae comitantes.

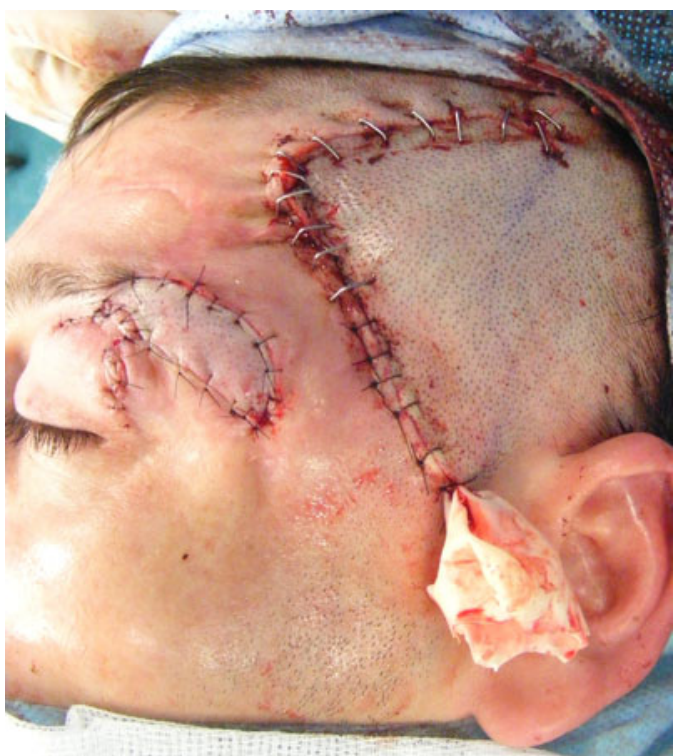

FIG. 3. First surgical stage intra-operative view: hair-bearing skin flap transferred to left brow and donor site repaired with a local rotation scalp flap.

struction: full-thickness scalp and eyebrow grafts, mini- or micro-scalp hair grafts, flaps from a thick ipsilateral or contralateral eyebrow, and axial skin island flap from the temporal scalp. ${ }^{1-4}$ Each technique has its advantages and disadvantages. A full-thickness hair-bearing skin graft, from the scalp or the eyebrow, is a time-honored technique that may undergo an unpredictable rate of take; within such a category a scalp skin graft provides a scalp-like high hair density; mini- or micro-hair grafts are an effective method of eyebrow restoration in terms of hair density and orientation,

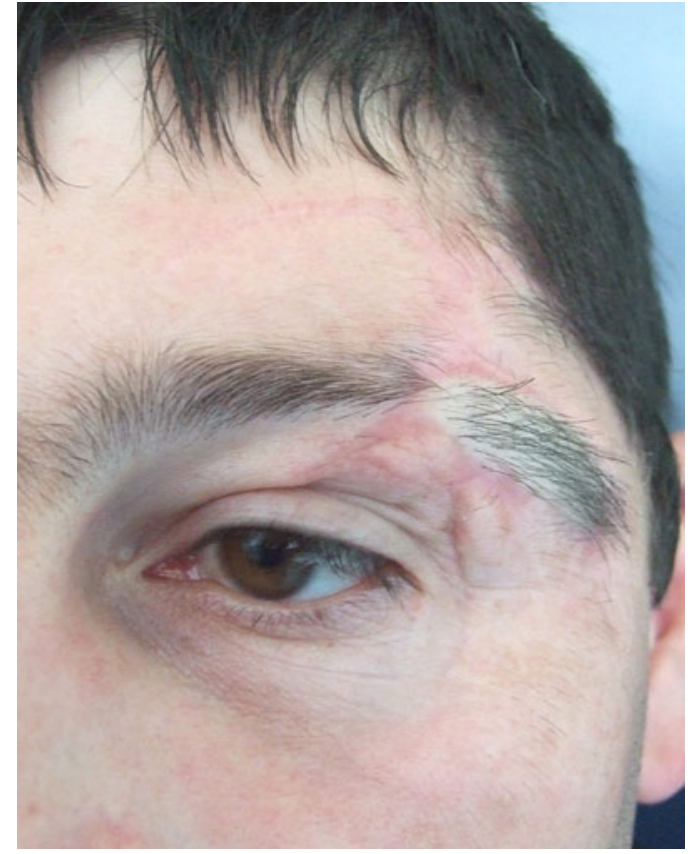

FIG. 4. First surgical stage postoperative view. 


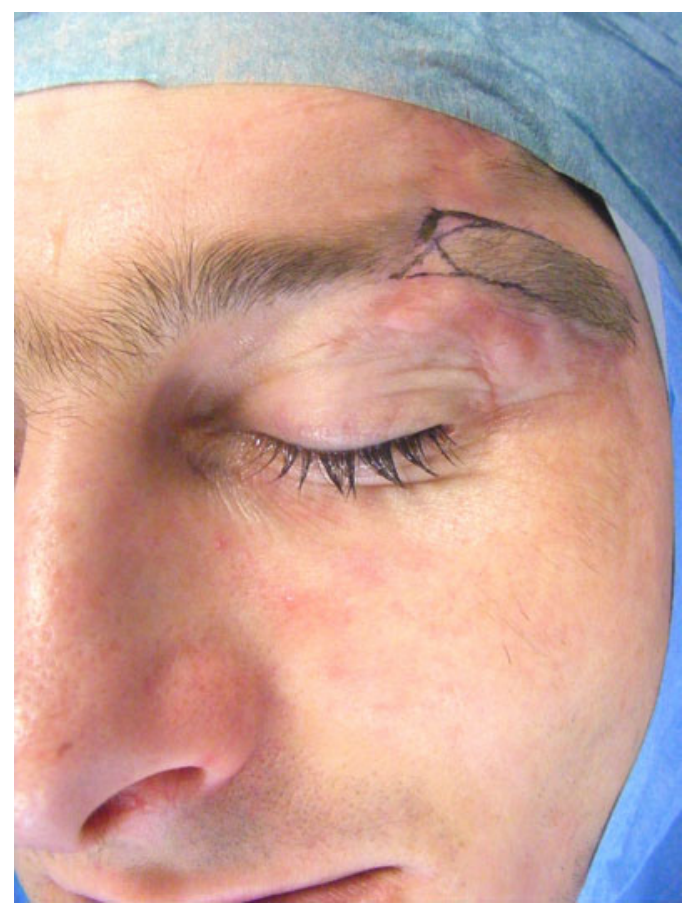

FIG. 5. Second surgical stage operative planning.

but are multi-stage and time-consuming procedures; ${ }^{1,2}$ the hair-bearing skin island eyebrow advancement flap is the appropriate solution for restoring the missing medial portion of the brow from a thick lateral one; ${ }^{3}$ the hair-bearing transposition flap from the contralateral eyebrow provides reconstruction with an excellent hair density, but with the wrong orientation, and a subsequent unnatural appearance; ${ }^{4}$ the axial skin island flap from the temporal scalp based on the anterior branch of the superficial temporal artery and venae comitantes provides a single-stage procedure with the correct hair orientation but with a scalp-like hair density. IPL is a very versatile tool that proved its effectiveness in a variety of skin conditions: hypertrichosis and hirsutism, skin

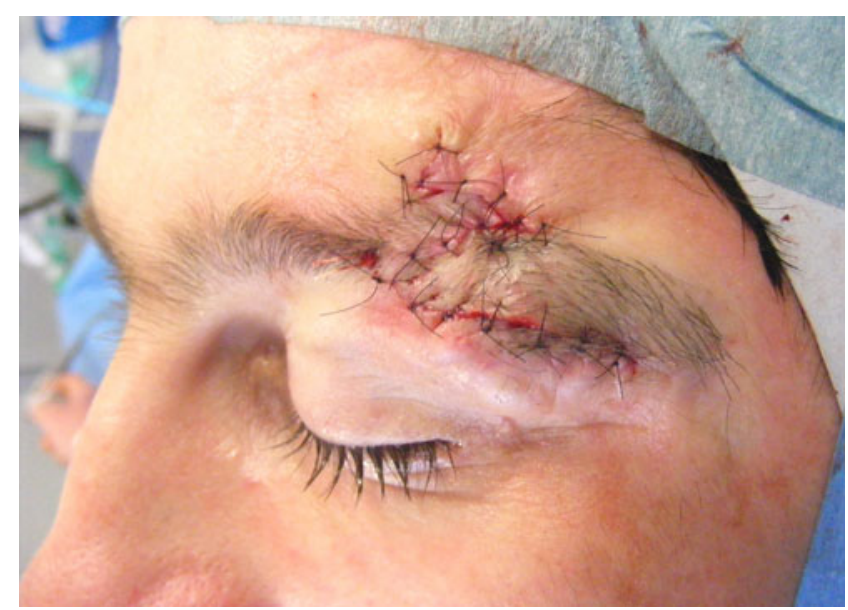

FIG. 6. Second surgical stage intra-operative view: multiple Z-plasties to refine alignment between the native medial eyebrow and the scalp hair-bearing island flap.

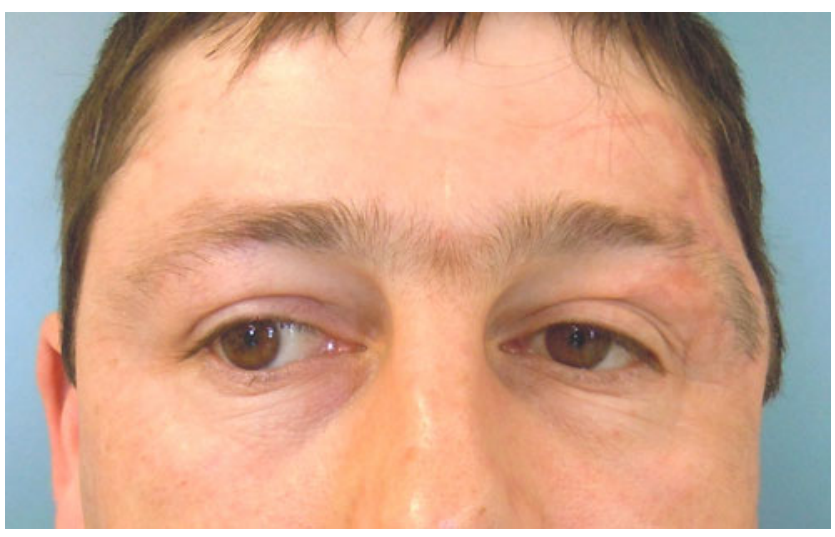

FIG. 7. Second surgical stage postoperative view.

vascular lesions (port wine stains, rosacea, teleangectasias, erythrosis), acne, and skin aging. ${ }^{5-13}$ The potential combination of different wavelengths, fluences, pulse durations, and pulse intervals allows a patient customized treatment within a wide spectrum of therapeutic indications. In our case, we deliberately preferred IPL to laser for hair removal, as its lesser hair count reduction could provide a smooth hair thinning out rather than an aggressive and more radical epilation in the reconstructed eyebrow. An increasing number of reports demonstrated the even superior effectiveness of IPL versus laser in the treatment of skin vascular alterations such as rosacea and erythrosis. $7,10,12$ Therefore, it was our option of choice for the treatment of persistent localized erythema in our patient's reconstructed eyebrow. Furthermore, to all of the previously mentioned features were added the demonstrated advantages of IPL in providing lower pain scores and local inflammation. $5,9,12$

IPL treatment was the only way to overcome the limitations of the traditional surgical technique, by providing a naturally irregular hair density in the reconstructed eyebrow.

Our experience demonstrated the efficacy of the integrated multidisciplinary approach involving traditional plastic reconstructive surgery and the innovative new technologies ${ }^{5-13}$ in aiming for perfection.

\section{Acknowledgments}

The authors thank Alan Serge McGhee, Glasgow City Council Education Department, for his contribution to the submission of this article.

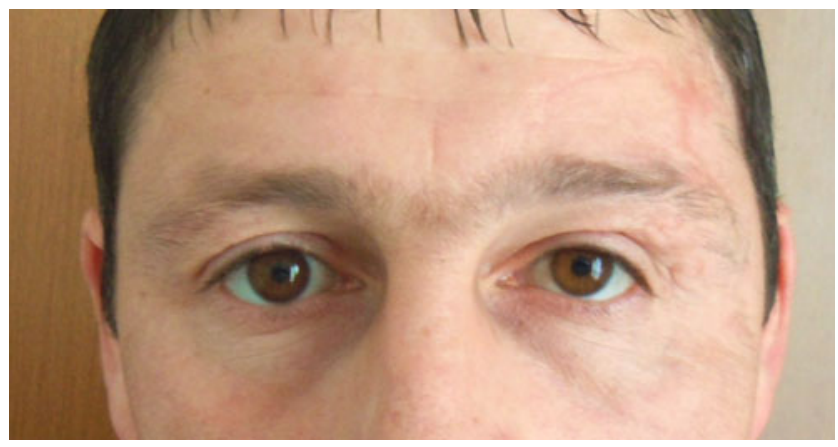

FIG. 8. Post- intense pulsed light (IPL) treatment view. 


\section{Author Disclosure Statement}

No competing financial interests exist.

\section{References}

1. Nordström, R.E. (1977). Eyebrow reconstruction by punch hair transplantation. Plast. Reconstr. Surg. 60, 74-76.

2. Rose, P.T. (2011). The latest innovations in hair transplantation. Facial Plast. Surg. 27, 366-377.

3. Bakkour, W., and Ghura, V. (2013). Medial eyebrow defects: reconstruction with whole eyebrow subcutaneous island pedicle Ophthal. Plast. Reconstr. Surg. 29, 330-332.

4. Mustardé, J.C. (1969). The eyebrows, in: Repair and Reconstruction in the Orbital Region. A Practical Guide. E. \& S. Livingstone: Edinburgh and London: pp. 247-248.

5. Cameron, H., Ibbotson, S.H., Dawe, R.S., Ferguson, J., and Moseley, J. (2008). Within-patients right-left blinded comparison of diode $(810 \mathrm{~nm})$ laser therapy and intense pulsed light therapy for hair removal. Lasers Med. Sci. 23, 393397.

6. Bahmer, F., Drosner, M., Hohenleutner, U., et al (2007). Recommendation for laser and intense pulsed light (IPL) therapy in dermatology. J. Dtsch. Dermatol. Ges. 5, 10361042.

7. Weiss, R.A., Ross, E.V., Tanghetti, E.A., et al (2011). Characterization of an optimized light source and comparison to pulsed dye laser for superficial and deep vessel clearance. Lasers Surg. Med. 43, 92-98.

8. Haedersdal, M., and Wulf, H.C. (2006). Evidence-based review of hair removal using lasers and light sources. J. Eur. Acad. Dermatol. Venereol. 20, 9-20.
9. Sochor, M., Curkova, A.K., Schwarczova, Z., Sochorova, R., Simaljakova, M., and Buchvald, J. (2011). Comparison of hair reduction with three lasers and light sources: prospective, blinded and controlled study. J. Cosmet. Laser Ther. 13, 210215.

10. Campolmi, P., Bonan, P., Cannarozzo, G., et al (2011). Intense pulsed light in the treatment of non-aesthetic facial and neck vascular lesions: report of 85 cases. J. Eur. Acad. Dermatol. Venereol. 25, 68-73.

11. Oh, S.H., Bae, B.K., Kim, T.G. et al (2010). Effective treatment of facial redness caused by atopic dermatitis using intense pulsed light systems. Dermatol. Surg. 36, 475-482.

12. Babilas, P., Schreml, S., Szeimies, R.M., and Landthaler, M. (2010). Intense pulsed light (IPL): a review. Lasers Surg. Med. 42, 93-104.

13. Nymann, P., Hedelund, L., and Haedersdal, M. (2010). Long-pulsed dye laser vs. intense pulsed light for the treatment of facial telangiectasias: a randomized controlled trial. J. Eur. Acad. Dermatol. Venereol. 24, 143-146.

Address correspondence to: Giovanni Nicoletti University of Pavia Salvatore Maugeri Research and Care Institute Via Salvatore Maugeri, 10 27100 Pavia Italy

E-mail: giovanni.nicoletti@unipv.it 